\title{
Promotion of metastasis of thyroid cancer cells via NRP-2-mediated induction
}

\author{
DOM-GENE TU ${ }^{1-3^{*}}$, WEN-WEI CHANG ${ }^{4 *}$, MING-SHIOU JAN $^{5-7^{*}}$, \\ CHI-WEN TU ${ }^{8}$, YIN-CHE LU ${ }^{9 *}$ and CHIEN-KUO TAI ${ }^{10}$ \\ ${ }^{1}$ Department of Nuclear Medicine, Ditmanson Medical Foundation, Chia-Yi Christian Hospital, Chia-Yi 600; \\ ${ }^{2}$ Department of Food Science and Technology, Chia Nan University of Pharmacy and Science, Tainan 717; \\ ${ }^{3}$ College of Health Sciences, Chang Jung Christian University, Tainan 711; ${ }^{4}$ School of Biomedical Sciences, \\ College of Medical Science and Technology; ${ }^{5}$ Immunology Research Center; ${ }^{6}$ Institute of Biochemistry, Microbiology \\ and Immunology, Chung Shan Medical University, Taichung 402; ${ }^{7}$ Division of Allergy, Immunology and Rheumatology; \\ Departments of ${ }^{8}$ Surgery and ${ }^{9}$ Hematology-Oncology, Ditmanson Medical Foundation, Chia-Yi Christian Hospital, \\ Chia-Yi 600; ${ }^{10}$ Department of Life Science, Institutes of Molecular Biology and Biomedical Science, \\ National Chung Cheng University, Min-Hsiung, Chia-Yi 621, Taiwan, R.O.C.
}

Received April 9, 2015; Accepted June 8, 2016

DOI: $10.3892 / \mathrm{ol} .2016 .5153$

\begin{abstract}
Tumor-node-metastasis is one of the leading causes of morbidity and mortality in thyroid cancer patients. Upregulation of vascular endothelial growth factor-C (VEGF-C) increases the migratory ability of thyroid cancer cells to lymph nodes. Expression of neuropilin-2 (NRP-2), the co-receptor of VEGF-C, has been reported to be correlated with lymph node metastasis in human thyroid cancer. The present study investigated the role of VEGF-C/NRP-2 signaling in the regulation of metastasis of two different types of human thyroid cancer cells. The results indicated that the VEGF-C/NRP-2 axis significantly promoted the metastatic activities of papillary thyroid carcinoma cells through the activation of the mitogen-activated protein kinase (MAPK) kinase (MEK)/extracellular signal-regulated kinase and p38 MAPK signaling cascades. However, neither MEK or p38 MAPK inhibitors produced significant inhibition of the migratory activity and invasiveness regulated by the VEGF-C/NRP-2 axis in follicular thyroid carcinoma cells. Finally, VEGF-C/NRP-2-mediated invasion
\end{abstract}

Correspondence to: Professor Chien-Kuo Tai, Department of Life Science, Institutes of Molecular Biology and Biomedical Science, National Chung Cheng University, No. 168, Sec. 1, University Road, Min-Hsiung, Chia-Yi 621, Taiwan, R.O.C.

E-mail: biockt@ccu.edu.tw

Abbreviations: VEGF-C, vascular endothelial growth factor-C; NRP-2, neuropilin-2; PTC, papillary thyroid carcinoma; FTC, follicular thyroid carcinoma

${ }^{*}$ Contributed equally

Key words: NRP-2, VEGF-C, thyroid cancer, tumor metastasis, lymphangiogenesis and migration of thyroid cancer cells required the expression of NRP-2. The present results demonstrate that the promotion of metastasis by VEGF-C is mainly due to the upregulation of NRP-2 in thyroid cancer cells, and this metastatic activity regulated by the VEGF-C/NRP-2 axis provides further insight into the process of tumor metastasis.

\section{Introduction}

The formation of metastasis requires multiple complex processes, including local invasion by cancer cells into lymphatic and blood vessels, trafficking and extravasation of cancer cells to the lymph node and distant organs, and development of small tumor nodules (1). Various factors and signaling networks enhance the metastatic potential of cancer cells by allowing tumor cells to survive, proliferate and migrate (1). Vascular endothelial growth factor-C (VEGF-C), a member of the VEGF family, has been reported to promote tumor lymphangiogenesis (2). VEGF-C is a secreted homodimeric glycoprotein with a central VEGF homology domain containing receptor binding sites for VEGF receptor-2 (VEGFR-2) and VEGFR-3 (2). Transgenic overexpression of VEGF-C in keratocytes causes lymphatic, but not vascular, endothelial proliferation and vessel enlargement in mouse skin (3). Several studies have demonstrated that cancers with lymph node metastasis usually express more VEGF-C than the corresponding normal mucosa (4), but this correlation is not universal (5), since VEGF-C expression is upregulated in numerous, but not all, human cancers $(6,7)$.

Neuropilin-2 (NRP-2) has been reported to function as a co-receptor for class 3 semaphorins and several VEGFs, including VEGF-C (8). NRP-2, a cell surface glycoprotein, is crucial for repulsive axon guidance, vascularization and angiogenesis $(9,10)$. Since it lacks intracellular signaling motifs, NRP-2 forms co-receptor complexes with plexins and VEGFRs such as VEGFR-3 to mediate signal transduction $(8,11)$. 
The role of NRP-2 in tumor pathogenesis has not been completely clarified. Expression of NRP-2 has been detected in breast (12) and pancreatic cancer (13). Furthermore, the expression levels of NRP-2 in lung lesions increased from dysplasia to microinvasive carcinoma (14). In addition, non-small-cell lung carcinoma patients with upregulated NRP-2 expression had a significantly worse prognosis than those without expression of NRP-2 (15).

Thyroid carcinoma is the most frequent malignancy of the endocrine system, mainly affecting women, with an estimated 60,220 new cases and 1,850 mortalities in the USA in 2013 (16). The majority of thyroid tumors follow an indolent clinical course with favorable prognosis (17). However, thyroid carcinoma has a tendency to spread into lymphatic channels and metastasize to regional lymph nodes at a high frequency (17-19). Vascular invasion may be an adverse prognostic sign, and thyroid cancer cells can metastasize via the bloodstream or the lymphatic vasculature $(18,19)$.

The mechanisms that determine the route of metastatic spread remain largely unknown. Several studies have reported that the expression of VEGF-C is correlated with metastasis in papillary thyroid carcinoma (PTC) $(18,19)$. These results indicate that VEGF-C expression may play a role in lymphangiogenesis of thyroid carcinoma and participate in the molecular regulation of tumor metastasis. In addition, NRP-2 expression was observed in $64.3 \%$ of PTC patients (20), and was reported to correlate with lymph node metastasis and VEGF-D expression in human PTC tissues (20). These data indicate that NRP-2 may contribute to the regulation of invasion and motility of thyroid cancer cells. Taken together, these studies suggest that VEGF-C and its co-receptor NRP-2 may be involved in the regulation of the metastatic potential of thyroid cancer cells.

In the present study, the role of VEGF-C/NRP-2 signaling in metastasis was characterized in two types of thyroid cancer cells, including PTC and follicular thyroid carcinoma (FTC), which represent $>90 \%$ of all thyroid malignancies (21). The results demonstrate that the activation of the VEGF-C/NRP-2 axis is mediated at least through the mitogen-activated protein kinase (MAPK) kinase (MEK)/extracellular signal-regulated kinase (ERK) and p38 MAPK signaling cascades in PTC cells. The VEGF-C/NRP-2 axis promotes the invasiveness and migration of thyroid cancer cells, and this axis critically requires NRP-2 for tumor invasion.

\section{Materials and methods}

Cell lines. The human K1 papillary thyroid cell line was purchased from the European Collection of Authenticated Cell Cultures (Salisbury, UK) and maintained in a 2:1:1 mixture medium of Dulbecco's modified Eagle's medium:Ham's F12: molecular cell developmental biology 105 medium (Sigma-Aldrich, St. Louis, MO, USA) supplemented with $10 \%$ (v/v) fetal bovine serum (FBS), $2 \mathrm{mM}$ glutamine, $100 \mathrm{IU} / \mathrm{ml}$ penicillin and $100 \mu \mathrm{g} / \mathrm{ml}$ streptomycin (Gibco; Thermo Fisher Scientific, Inc., Waltham, MA, USA). The human thyroid cancer WRO cell line (provided by Dr Jen-Der Lin, Chang Gung Memorial Hospital, Taoyuan, Taiwan) was cultured in RPMI medium (Gibco; Thermo Fisher Scientific, Inc.) supplemented with 10\% (v/v) FBS (Gibco; Thermo Fisher
Scientific, Inc.), $100 \mathrm{IU} / \mathrm{ml}$ penicillin and $100 \mu \mathrm{g} / \mathrm{ml}$ streptomycin (Gibco; Thermo Fisher Scientific, Inc.). Both cell lines were maintained at $37^{\circ} \mathrm{C}$ in a humidified $5 \% \mathrm{CO}_{2} / 95 \%$ air atmosphere.

Overexpression of NRP-2. Expression plasmid of human NRP-2 (GenBank: NM_201266; http://www.ncbi.nlm. nih.gov/nuccore/41872561) was purchased from OriGene Technologies, Inc. (Rockville, MD, USA). Transfection was performed using Gene $\operatorname{In}^{\mathrm{TM}}$ transfection reagent according to the manufacturer's protocol (MTI-GlobalStem, Gaithersburg, MD, USA). In brief, cells were seeded in 6-well culture plates $\left(0.5-1.0 \times 10^{6} \mathrm{cells} / \mathrm{ml} /\right.$ well $)$ and transfected with $8 \mu \mathrm{g}$ expression plasmid for $36 \mathrm{~h}$. Transfected cells were starved in $4 \%(\mathrm{v} / \mathrm{v})$ FBS medium for the next $16 \mathrm{~h}$. To stimulate the VEGF-C/NRP-2 axis, the cells were treated with $100 \mathrm{ng} / \mathrm{ml}$ human recombinant VEGF-C (PeproTech Inc., Rocky Hill, NJ, USA) for the indicated times. To block the VEGF-C/NRP-2 interaction, the cells were pre-incubated with an NRP-2 function-blocking antibody $(0.2 \mu \mathrm{g} / \mathrm{ml}$; R\&D Systems, Inc., Minneapolis, MN, USA) for $1.5 \mathrm{~h}$ before being stimulated with VEGF-C (22). To determine the signaling pathways, the transfected cells were pre-incubated with PD98059 $(25 \mu \mathrm{M})$, SB203580 $(10 \mu \mathrm{M})$ or SB202190 $(20 \mu \mathrm{M})$ (Sigma-Aldrich) alone for $0.5 \mathrm{~h}$, followed by VEGF-C stimulation, as mentioned above. After incubation, the cells were collected and subjected to western blot analysis as described previously (23). The antibodies used were directed against the Myc epitope tag (cat. no. 2276; 1:1,000), phosphorylated (p)-ERK (cat. no. 4376; 1:2,000), ERK (cat. no. 4695; 1:2,000), p-p38 MAPK (cat. no. 9215; 1:2,000) and p38 MAPK (cat. no. 9212; 1:2,000) (Cell Signaling Technology, Inc., Danvers, MA, USA). All assays were performed in triplicate.

Migration and invasion assays. To analyze cell migration using in vitro scratch assay, NRP-2-overexpressing cells were seeded in triplicate in 24-well plates $\left(1.5-4.0 \times 10^{5}\right.$ cells $\left./ \mathrm{ml} / \mathrm{well}\right)$ and cultured in $4 \%$ (v/v) FBS medium overnight at $37^{\circ} \mathrm{C}(24,25)$. Upon washing with phosphate-buffered saline, the cell monolayer was scraped in a straight line with a pipette tip. After incubation with culture medium (4\% FBS) containing VEGF-C plus or minus PD98059, SB203580 or SB202190 for $8 \mathrm{~h}$, the cells were observed using a Nikon inverted microscope (Nikon Corporation, Tokyo, Japan) and Image Pro-Plus image analysis software (Media Cybernetics, Inc., Rockville, MD, USA). Images were obtained of $\geq 4$ randomly selected microscopic fields per well, and four randomly selected gaps were calculated per photograph. The invasive activity of NRP-2-overexpressing cells was examined using the Corning ${ }^{\circledR}$ BioCoat $^{\mathrm{TM}}$ Tumor Invasion System (Corning Life Sciences, Tewksbury, MA, USA) according to the manufacturer's protocol (25). In brief, $5 \times 10^{4}$ cells were resuspended in $200 \mu \mathrm{l} 4 \% \mathrm{FBS}$ medium and placed in the top chamber (8- $\mu \mathrm{m}$ insert) in triplicate wells for each group (VEGF-C plus or minus PD98059, SB203580 or SB202190), while the lower chamber was coated with Matrigel. After incubation for $24 \mathrm{~h}$, the cells from the top chamber were removed using a cotton swab, and the cells on the lower surface of the insert were fixed and stained using Giemsa stain (Sigma-Aldrich). The number of cells was counted using a Nikon inverted 
A

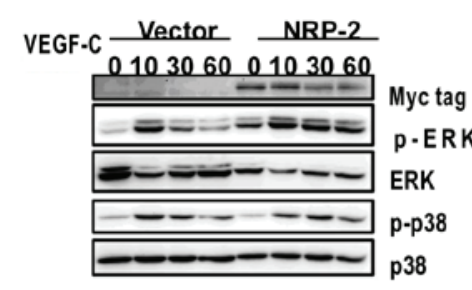

D

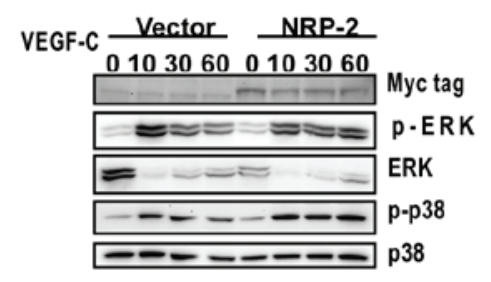

B

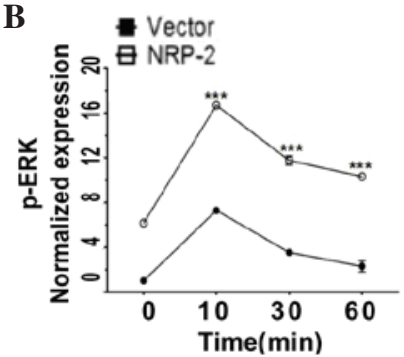

E

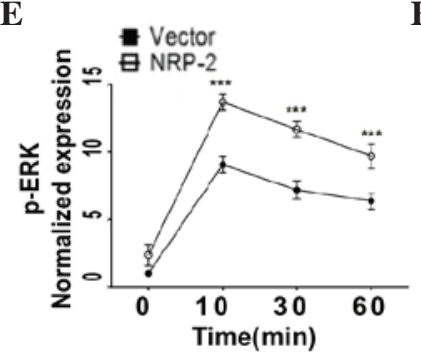

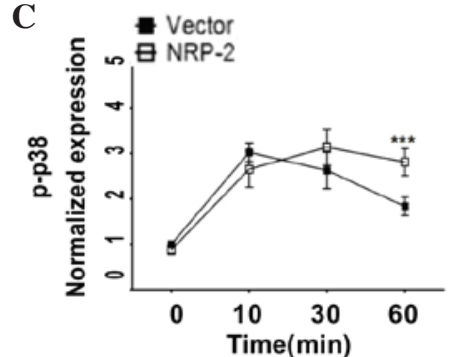

F

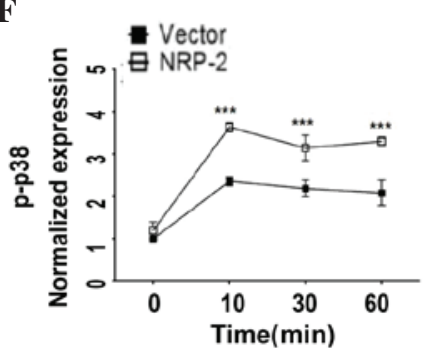

Figure 1. Sequential phosphorylation of ERK and p38 MAPK in response to VEGF-C stimulation in NRP-2-overexpressing thyroid cancer cells. NRP-2-overexpressing (A-C) K1 and (D-F) WRO cells were treated with $100 \mathrm{ng} / \mathrm{ml}$ VEGF-C at different time intervals, as indicated. (A) The levels of p-ERK and p-p38 MAPK in K1 cells were measured using western blot analysis. An anti-Myc tag antibody was used to detect the expression of NRP-2. Representative western blots from three independent experiments are shown. (B and C) The results of the densitometric analysis from three independent western blot analyses are shown. (B) p-ERK/total-ERK and (C) p-p38 MAPK/total-p38 MAPK ratios in K1 cells were expressed as the relative variation vs. the basal levels of each single experiment. (D) The levels of p-ERK and p-p38 MAPK in WRO cells were measured using western blot analysis. An anti-Myc tag antibody was used to detect the expression of NRP-2. Representative western blots from three independent experiments are shown. (E and F) The results of the densitometric analysis from three independent western blot analyses are shown. (E) p-ERK/total-ERK and (F) p-p38 MAPK/total-p38 MAPK ratios in WRO cells were expressed as the relative variation vs. the basal levels of each single experiment. ${ }^{* * *} \mathrm{P}<0.001$. VEGF, vascular endothelial growth factor; NRP-2, neuropilin-2; MAPK, mitogen-activated protein kinase; ERK, extracellular signal-regulated kinase; p-, phosphorylated.

microscope (Nikon Corporation). All assays were performed in triplicate.

Statistical analysis. Data were expressed as the mean \pm standard deviation. Group comparisons were performed using analysis of variance with Tukey's comparison test. All statistical analysis was performed using GraphPad Prism software (version 6; GraphPad Software Inc., San Diego, CA, USA). P<0.05 was considered to indicate a statistically significant difference.

\section{Results}

The VEGF-C/NRP-2 axis activates ERK and p38 MAPK in thyroid cancer cells. In order to test the aforementioned hypothesis, the present study first examined whether NRP-2 could be expressed in different types of human thyroid cancer cells, including K1 (PTC) and WRO (FTC). As indicated in Fig. 1, overexpressed NRP-2, which had a C-terminal Myc tag, was detected as a single major band in the two thyroid cell lines.

Next, to study the VEGF-C/NRP-2 axis in the two thyroid cancer cells, NRP-2-overexpressing cells were treated with exogenous recombinant human VEGF-C, and the activities of p-ERK and p-p38 MAPK were evaluated. In both K1 and WRO cells, when NRP-2 was overexpressed, an early and transient activation of p-ERK was observed following VEGF-C induction $(100 \mathrm{ng} / \mathrm{ml})$, which peaked at $10 \mathrm{~min}$ [2.29-fold for the K1 cells (Fig.1A and B) and 1.51-fold for the WRO cells (Fig.1D and E)] and remained elevated at $60 \mathrm{~min}$ compared with the levels displayed by the vector-only-transfected cells. Similarly, VEGF-C strongly activated p-p38 MAPK, which was maximal at $10 \mathrm{~min}$ (1.54-fold for WRO cells) and was sustained for $\leq 60 \mathrm{~min}$ in the NRP-2-overexpressing WRO cells (Fig.1D and F), while p-p38 MAPK was markedly activated at 60 min in the NRP-2-overexpressing K1 cells compared with the levels detected in the vector only-transfected cells, which were declining toward basal levels (Fig. 1A and C). Consistent with previous studies reporting that NRP-2 functions as a co-receptor for VEGF (8), the present data demonstrated that the VEGF-C/NRP-2 axis is activated with differential kinetics of kinase activation in the two types of human thyroid cancer cells evaluated.

To further investigate the downstream pathways of the VEGF-C/NRP-2 axis in thyroid cancer cells, PD98059, a potent and selective inhibitor of MEK activation, and SB203580, a selective and potent inhibitor of p38 MAPK (26), were used in NRP-2-overexpressing cells (Fig. 2). As expected, treatment with PD98059 $(25 \mu \mathrm{M})$ strongly abolished the stimulated ERK-mediated activation of the VEGF-C/NRP-2 axis in K1 cells (Fig. 2A), but barely exerted any effect on the activated p-p38 (Fig. 2A). However, neither PD98059 nor SB203580 $(10 \mu \mathrm{M})$ produced any inhibition of ERK and p38 MAPK phosphorylation on the VEGF-C/NRP-2 axis in WRO cells (Fig. 2B and D). Unexpectedly, SB203580 inhibited both p38 MAPK and ERK phosphorylation on the VEGF-C/NRP-2 axis in $\mathrm{K} 1$ cells (Fig. 2C).

To ascertain the effect of p38 MAPK inhibitors on the VEGF-C/NRP-2 axis, another cell permeable inhibitor of p38 MAPK, SB202190 (26), was used in NRP-2-overexpressing cells. Similarly, the phosphorylation levels of p38 MAPK and ERK on the VEGF-C/NRP-2 axis were suppressed by SB202190 $(20 \mu \mathrm{M})$ in $\mathrm{K} 1$ cells (Fig. 2E), whereas the 

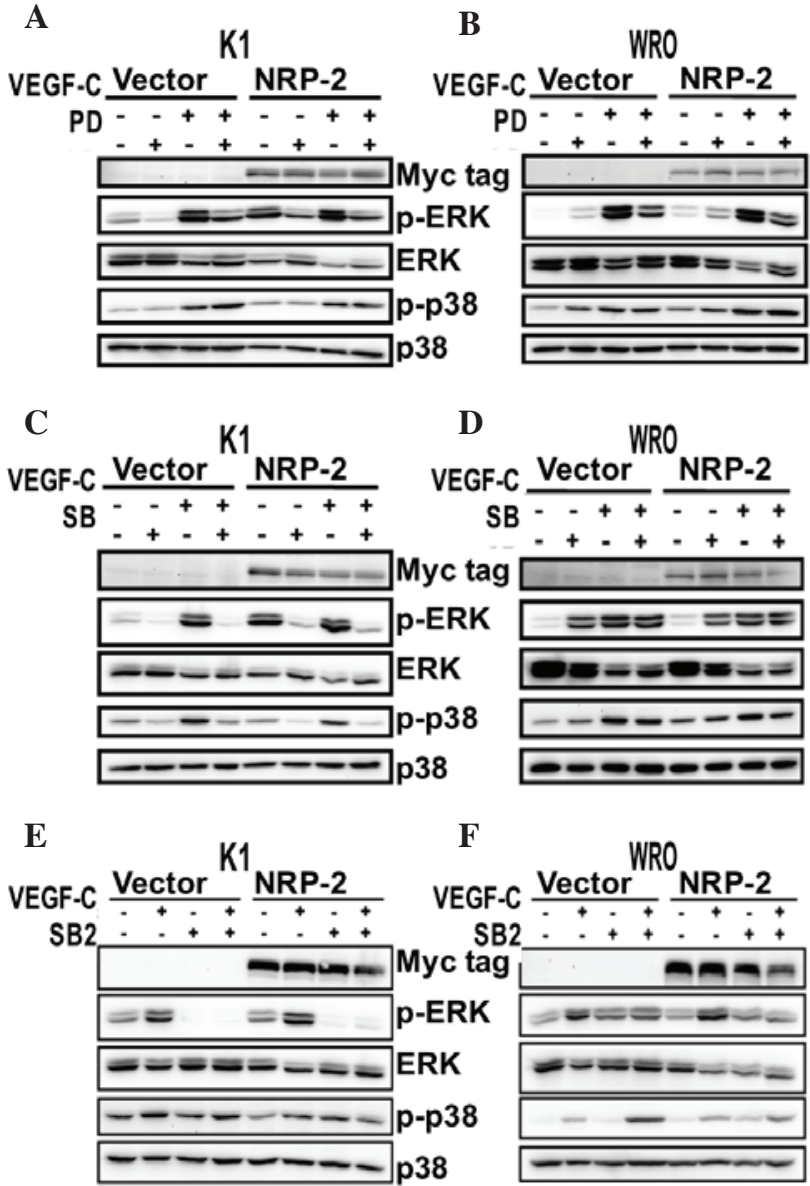

Figure 2. Effect of PD, SB and SB2 on the VEGF-C/NRP-2 axis NRP-2-overexpressing K1 and WRO cells were either pretreated with (A and B) PD $(25 \mu \mathrm{M}),(\mathrm{C}$ and D) SB $(10 \mu \mathrm{M}),(\mathrm{E}$ and F) SB2 $(20 \mu \mathrm{M})$ or DMSO for $30 \mathrm{~min}$, and then stimulated with VEGF-C $(100 \mathrm{ng} / \mathrm{ml})$ for $10 \mathrm{~min}$. The levels of p-ERK and p-p38 MAPK were measured using western blot analysis. An anti-Myc tag antibody was used to detect the expression of NRP-2. Representative western blots from three independent experiments are shown. VEGF, vascular endothelial growth factor; NRP-2, neuropilin-2; MAPK, mitogen-activated protein kinase; ERK, extracellular signal-regulated kinase; p-, phosphorylated; PD, PD98059; SB, SB203580; SB2, SB202190; DMSO, dimethyl sulfoxide.

phosphorylation levels of p38 MAPK and ERK were not affected by SB202190 in WRO cells (Fig. 2F).

Consistent with previous studies reporting that the activation of ERK is an important kinase cascade in the regulation of VEGF-C signaling in cancer cells $(27,28)$, the present data suggest that the activation of the VEGF-C/NRP-2 axis is mediated at least through the MEK/ERK and p38 MAPK signaling cascades in K1 cells. Furthermore, since the present results did not reveal significant differences in the treatment of WRO cells with inhibitors, other signaling cascades may be regulated by the VEGF-C/NRP-2 axis in WRO cells.

The VEGF-C/NRP-2 axis promotes migration and invasion of thyroid cancer cells. Wound-healing assay and in vitro invasion assay were used to examine the role of the VEGF-C/NRP-2 axis on the migration and invasiveness of thyroid cancer cells (Fig. 3). Treatment with VEGF-C significantly increased the migratory activities (Fig. 3A and $\mathrm{C}$ ) and invasiveness (Fig. 3B and D) of the two NRP-2-overexpressing thyroid
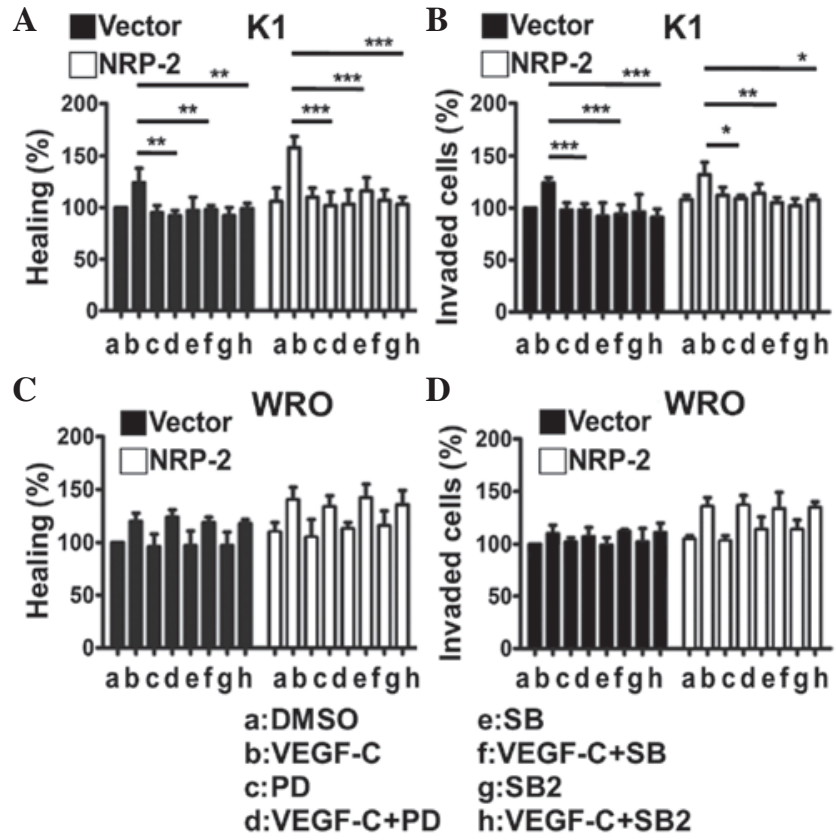

Figure 3. Effect of the inhibition of the VEGF-C/NRP-2 axis on the metastasis of thyroid cancer cells. NRP-2-overexpressing (A and B) K1 and (C and D) WRO cells were treated with VEGF-C $(100 \mathrm{ng} / \mathrm{ml})$ plus or minus PD98059, SB203580 or SB202190. Bar graphs represent the results from (A and $C$ ) scratch wound-healing assay (as the mean \pm SD of the relative percentages of the wounds gap width) and (B and D) invasion assay (as the mean \pm SD of the relative percentages of invading cells) for each treatment from three independent experiments. There was no significant difference in the suppression of VEGF-C/NRP-2-mediated metastasis in NRP-2-overexpressing WRO cells treated with inhibitors plus VEGF-C. ${ }^{*} \mathrm{P}<0.05 ;{ }^{* *} \mathrm{P}<0.01 ;{ }^{* * *} \mathrm{P}<0.001$. VEGF, vascular endothelial growth factor; NRP-2, neuropilin-2; PD, PD98059; SB, SB203580; SB2, SB202190; DMSO, dimethyl sulfoxide; SD, standard deviation.

cancer cells compared with vector-transfected cells $(\mathrm{P}<0.05)$. In addition, overexpression of NRP-2 did not significantly affect the migratory ability or invasiveness of the two thyroid cells compared with the vector-transfected cells (Fig. 3). These results indicate that the VEGF-C/NRP-2 axis can promote the invasiveness and migratory ability of thyroid cancer cells.

In $\mathrm{K} 1$ cells, western blot analysis revealed that the MEK/ERK and $\mathrm{p} 38$ MAPK signaling cascades were activated by the VEGF-C/NRP-2 axis (Fig. 2A-C). Accordingly, the invasive activities of NRP-2-overexpressing $\mathrm{K} 1$ cells were significantly suppressed by PD98059, SB203580 and SB202190 (Fig. 3B). Together with the results mentioned above, the present data suggest that the activation of the VEGF-C/NRP-2 axis is mediated at least through the MEK/ERK and p38 MAPK signaling cascades, and further regulates the invasive activities of K1 cells. As for WRO cells, the present results did not reveal significant differences in the treatment of cells with MEK or p38 MAPK inhibitors following the activation of the VEGF-C/NRP-2 axis (Fig. 2D-F). As a result, these inhibitors did not produce a significant inhibition of the migratory activity (Fig. 3C) or invasiveness (Fig. 3D) of NRP-2-overexpressing WRO cells, which provides additional evidence that other signaling cascades may be regulated by the VEGF-C/NRP-2 axis in WRO cells.

To further rule out the possibility that the effect of the VEGF-C/NRP-2 axis on invasiveness was caused by different 

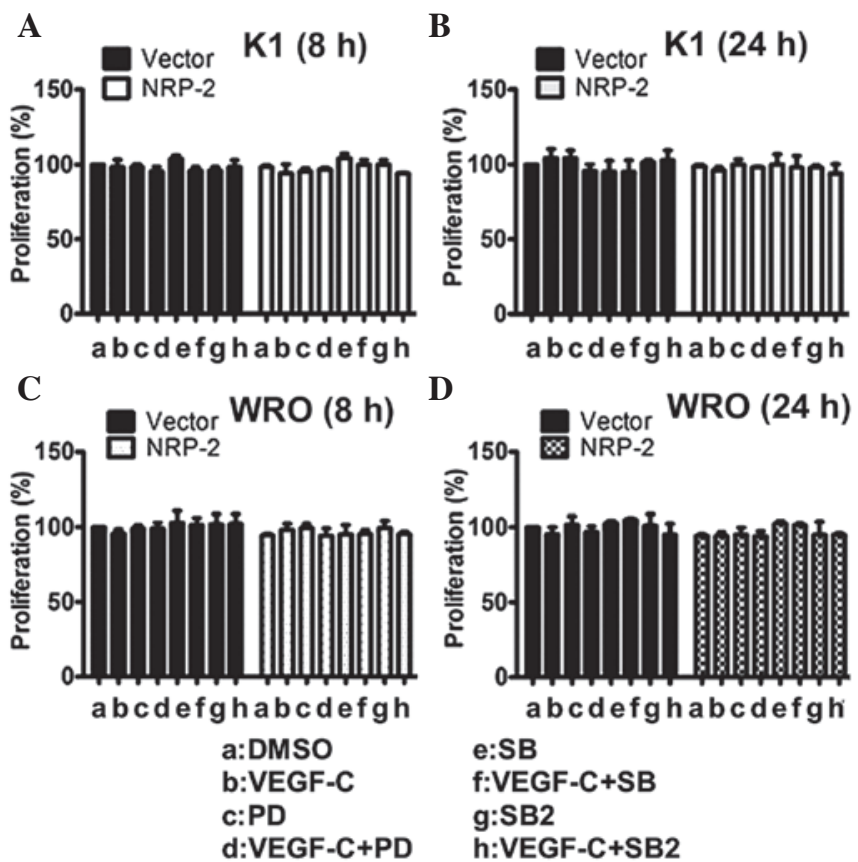

Figure 4. Effect of the VEGF-C/NRP-2 axis on cell proliferation of thyroid cancer cells. NRP-2-overexpressing (A and B) K1 and (C and D) WRO cells were treated with VEGF-C $(100 \mathrm{ng} / \mathrm{ml})$ plus or minus PD98059, SB203580 or SB202190 for (A and C) $8 \mathrm{~h}$ or (B and D) $24 \mathrm{~h}$. Bar graphs represent the mean \pm standard deviation of the relative percentages of cell proliferation for each treatment from three independent experiments. No significant differences were observed between the different treatments. VEGF, vascular endothelial growth factor; NRP-2, neuropilin-2; MAPK, mitogen-activated protein kinase; PD, PD98059; SB, SB203580; SB2, SB202190; DMSO, dimethyl sulfoxide.

proliferation rates, the growth rates of the NRP-2-overexpressing cells were compared (Fig. 4). The control cells and the NRP-2-overexpressing cells had similar growth rates after 8 (Fig. 4A and C) and $24 \mathrm{~h}$ (Fig. 4B and D) in culture. Therefore, these results indicate that the increased mobility of thyroid cancer cells was due to the activation of the VEGF-C/NRP-2 axis. Taken together, these data strongly support the finding that the VEGF-C/NRP-2 axis is actively involved in regulating the mobility and invasiveness of thyroid cancer cells.

VEGF-C/NRP-2 axis-mediated migration and invasion of thyroid cancer cells require NRP-2 signaling. To evaluate more rigorously the role of NRP-2 in the invasiveness of thyroid cancer cells following the activation of the VEGF-C/NRP-2 axis, an NRP-2 function-blocking antibody was used to selectively block the binding of the VEGF family ligands to NRP-2 $(29,30)$. In NRP-2-overexpressing K1 cells, the VEGF-C/NRP-2 axis became defective due to the reduction in ERK and p38 MAPK phosphorylation (Fig. 5A). Of note, the phosphorylation levels of ERK and p38 MAPK were significantly suppressed in the NRP-2-overexpressing WRO cells (Fig. 5B). Additionally, treatment with the NRP-2 function-blocking antibody in NRP-2-overexpressing thyroid cancer cells also strongly impaired VEGF-C/NRP-2-induced migratory activity (Fig. 5C) and invasiveness (Fig. 5D). Taken together, the present data suggest that the VEGF-C/NRP-2 axis is important in the invasiveness of thyroid cancer cells, and that this axis critically requires NRP-2 for cell invasion.
A
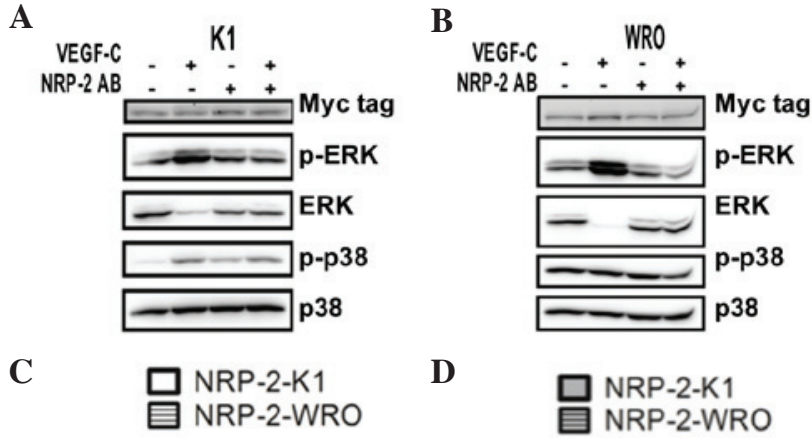

$\mathbf{D}$
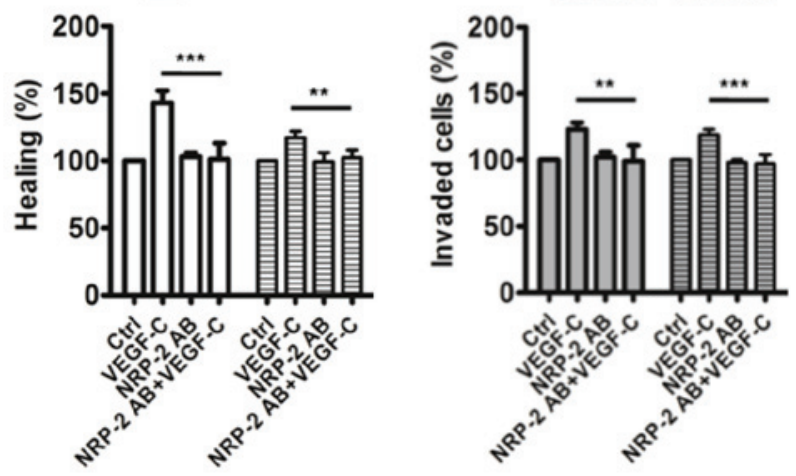

Figure 5. NRP-2 blocking signals suppress VEGF-C-induced metastasis NRP-2-overexpressing (A) K1 and (B) WRO cells were pretreated with an NRP-2 function-blocking antibody $(0.5 \mu \mathrm{g} / \mathrm{ml})$ for $1.5 \mathrm{~h}$, and then stimulated with VEGF-C (100 ng/ml) for $10 \mathrm{~min}$. The levels of extracellular signal-regulated kinase and p38 mitogen-activated protein kinase phosphorylation were measured using western blot analysis. An anti-Myc tag antibody was used to detect the expression of NRP-2. Representative western blots from three independent experiments are shown. (C) Scratch wound-healing assay and (D) invasion assay were performed to evaluate the effect of NRP-2 blocking signals on cell migration and invasion. Bar graphs represent the mean \pm standard deviation of the relative percentages for each treatment from three independent experiments. ${ }^{* *} \mathrm{P}<0.01 ;{ }^{* * * *} \mathrm{P}<0.001$. VEGF, vascular endothelial growth factor; NRP-2, neuropilin-2; MAPK, mitogen-activated protein kinase; ERK, extracellular signal-regulated kinase; p-, phosphorylated; PD, PD98059; SB, SB203580; SB2, SB202190; Ctrl, control; AB, antibody.

\section{Discussion}

As previously reported, regulation of tumor metastasis by VEGF-C occurs by increasing the migratory ability of cancer cells to lymph nodes (29). NRP-2 has been documented to be a co-receptor for VEGF-C (8), and lymph node metastasis of human PTC has been correlated to NRP-2 expression (12). However, the roles of NRP-2 and its ligand, VEGF-C, in the metastasis of thyroid cancer cells remain largely unknown. In the present study, the activation of the VEGF-C/NRP-2 axis was mediated at least through the MEK/ERK and p38 MAPK signaling cascades, particularly in human PTC (K1) cells. The migratory activity and invasiveness of thyroid cancer cells were further upregulated by the activation of the VEGF-C/NRP-2 axis, and this cell invasion mediated by the VEGF-C/NRP-2 axis was observed to be NRP-2 dependent.

To the best of our knowledge, the present study is the only detailed report on the effect of the VEGF-C/NRP-2 axis on the migratory activities of follicular thyroid cell-derived tumors, including PTC and FTC. The present report emphasizes that the presence of VEGF-C has a complex association with clinicopathological factors such as NRP-2 in the metastasis of thyroid cancer. Indeed, NRP-2 signaling has been reported to contribute 
to focal adhesion kinase (FAK)-mediated signaling cascade activation, and is further involved in the initiation of tumorigenesis (30). Inhibition of FAK activation also resulted in the suppression of proliferation and migration of breast cancer cells in vitro (31). These studies suggest that FAK activation may be involved in the metastasis of thyroid cancer through the regulation of the VEGF-C/NRP-2 axis. However, further experiments are required to address this possibility.

Activation of nuclear factor- $\kappa \mathrm{B}(\mathrm{NF}-\kappa \mathrm{B})$ has been demonstrated to be involved in cell proliferation, resistance to apoptosis, and promotion of tumor angiogenesis and metastasis, including those reported in human FTC (32). In the present study, neither MEK or p38 MAPK inhibitors exhibited a significant inhibition of migratory activity and invasiveness in WRO FTC cells. A possible explanation for this observation is that the VEGF-C/NRP-2 axis activates not only the MEK/ERK and p38 MAPK signaling cascades, but also the NF- $\mathrm{B}$ signaling cascade. Since $\mathrm{NF}-\kappa \mathrm{B}$ activation was increased in VEGF or NRP-mediated cell migration $(33,34)$, whether activation of $\mathrm{NF}-\kappa \mathrm{B}$ also contributes to VEGF-C/NRP-2 axis-mediated cell migration requires further investigation.

The present study revealed that SB203580 and SB202190 inhibited both ERK and p38 MAPK phosphorylation on the VEGF-C/NRP-2 axis in K1 cells. The proto-oncogene c-Raf, an upstream serine/threonine kinase of the MEK/ERK signaling cascade, was reported to be inhibited by SB203580 in vitro (35). Whether the activation of c-Raf is affected by the VEGF-C/NRP-2 axis in thyroid cancer cells remains to be determined.

Several studies have reported that the expression of VEGF-C protein and its messenger RNA are correlated with metastasis in PTC $(18,19)$. These reports suggest that other factors are likely to be involved in the regulation of VEGF-C-mediated metastatic status of thyroid carcinomas. In the present study, it was observed that NRP-2 was required for the VEGF-C/NRP-2 axis to promote cell migration and invasiveness of thyroid cancer cells. In fact, NRP-2 expression was previously reported to be correlated with VEGF expression and lymph node status in PTC (20). Furthermore, the serum levels of VEGF factors were reported to be promising diagnostic tools in patients with lung cancer $(36,37)$. These previous studies and the present findings suggest that NRP-2 and VEGF-C may act as key analytic markers for thyroid cancer metastases and prognosis. Future experiments are required to evaluate the clinical application of NRP-2 and VEGF-C expression in human thyroid cancer specimens.

\section{Acknowledgements}

The present study was supported by the Ditmanson Medical Foundation of Chia-Yi Christian Hospital (Chia-Yi, Taiwan; grant no. CSMU-CYC-102-02).

\section{References}

1. Fidler IJ: The pathogenesis of cancer metastasis: The 'seed and soil' hypothesis revisited. Nat Rev Cancer 3: 453-458, 2003.

2. Joukov V, Pajusola K, Kaipainen A, Chilov D, Lahtinen I, Kukk E, Saksela O, Kalkkinen N and Alitalo K: A novel vascular endothelial growth factor, VEGF-C, is a ligand for the Flt4 (VEGFR-3) and KDR (VEGFR-2) receptor tyrosine kinases. EMBO J 15: 290-298, 1996.
3. Jeltsch M, Kaipainen A, Joukov V, Meng X, Lakso M, Rauvala H, Swartz M, Fukumura D, Jain RK and Alitalo K: Hyperplasia of lymphatic vessels in VEGF-C transgenic mice. Science 276: 1423-1425, 1997.

4. Amioka T, Kitadai Y, Tanaka S, Haruma K, Yoshihara M, Yasui $\mathrm{W}$ and Chayama K: Vascular endothelial growth factor-C expression predicts lymph node metastasis of human gastric carcinomas invading the submucosa. Eur J Cancer 38: 1413-1419, 2002.

5. George ML, Tutton MG, Janssen F, Arnaout A, Abulafi AM, Eccles SA and Swift RI: VEGF-A, VEGF-C, and VEGF-D in colorectal cancer progression. Neoplasia 3: 420-427, 2001.

6. Niki T, Iba S, Tokunou M, Yamada T, Matsuno Y and Hirohashi S: Expression of vascular endothelial growth factors A, B, C, and D and their relationships to lymph node status in lung adenocarcinoma. Clin Cancer Res 6: 2431-2439, 2000.

7. Gunningham SP, Currie MJ, Han C, Robinson BA, Scott PA, Harris AL and Fox SB: The short form of the alternatively spliced flt- 4 but not its ligand vascular endothelial growth factor $\mathrm{C}$ is related to lymph node metastasis in human breast cancers. Clin Cancer Res 6: 4278-4286, 2000.

8. Favier B, Alam A, Barron P, Bonnin J, Laboudie P, Fons P, Mandron M, Herault JP, Neufeld G, Savi P, et al: Neuropilin-2 interacts with VEGFR-2 and VEGFR-3 and promotes human endothelial cell survival and migration. Blood 108: 1243-1250, 2006.

9. Giger RJ, Cloutier JF, Sahay A, Prinjha RK, Levengood DV, Moore SE, Pickering S, Simmons D, Rastan S, Walsh FS, et al: Neuropilin-2 is required in vivo for selective axon guidance responses to secreted semaphorins. Neuron 25: 29-41, 2000.

10. Yuan L, Moyon D, Pardanaud L, Bréant C, Karkkainen MJ, Alitalo K and Eichmann A: Abnormal lymphatic vessel development in neuropilin 2 mutant mice. Development 129: 4797-4806, 2002.

11. Pellet-Many C, Frankel P, Jia H and Zachary I: Neuropilins: Structure, function and role in disease. Biochem J 411: 211-226, 2008.

12. Yasuoka H, Kodama R, Tsujimoto M, Yoshidome K, Akamatsu H, Nakahara M, Inagaki M, Sanke T and Nakamura Y: Neuropilin-2 expression in breast cancer: Correlation with lymph node metastasis, poor prognosis, and regulation of CXCR4 expression. BMC Cancer 9: 220, 2009.

13. Cohen T, Herzog Y, Brodzky A, Greenson JK, Eldar S, Gluzman-Poltorak Z, Neufeld G and Resnick MB: Neuropilin-2 is a novel marker expressed in pancreatic islet cells and endocrine pancreatic tumours. J Pathol 198: 77-82, 2002.

14. Lantuéjoul S, Constantin B, Drabkin H, Brambilla C, Roche J and Brambilla E: Expression of VEGF, semaphorin SEMA3F, and their common receptors neuropilins NP1 and NP2 in preinvasive bronchial lesions, lung tumours, and cell lines. J Pathol 200: 336-347, 2003.

15. Kawakami T, Tokunaga T, Hatanaka H, Kijima H, Yamazaki H, Abe Y, Osamura Y, Inoue H, Ueyama Y and Nakamura M: Neuropilin 1 and neuropilin 2 co-expression is significantly correlated with increased vascularity and poor prognosis in nonsmall cell lung carcinoma. Cancer 95: 2196-2201, 2002.

16. Siegel R, Naishadham D and Jemal A: Cancer statistics, 2013. CA Cancer J Clin 63: 11-30, 2013.

17. Lang BH, Lo CY, Chan WF, Lam KY and Wan KY: Prognostic factors in papillary and follicular thyroid carcinoma: Their implications for cancer staging. Ann Surg Oncol 14: 730-738, 2007.

18. Salajegheh A, Pakneshan S, Rahman A, Dolan-Evans E, Zhang S, Kwong E, Gopalan V, Lo CY, Smith RA and Lam AK: Co-regulatory potential of vascular endothelial growth factor-A and vascular endothelial growth factor- $\mathrm{C}$ in thyroid carcinoma. Hum Pathol 44: 2204-2212, 2013.

19. Yu XM, Lo CY, Lam AK, Lang BH, Leung P and Luk JM: The potential clinical relevance of serum vascular endothelial growth factor (VEGF) and VEGF-C in recurrent papillary thyroid carcinoma. Surgery 144: 934-940; discussion 940-941, 2008.

20. Yasuoka H, Kodama R, Hirokawa M, Takamura Y, Miyauchi A, Inagaki M, Sanke T and Nakamura Y: Neuropilin-2 expression in papillary thyroid carcinoma: Correlation with VEGF-D expression, lymph node metastasis, and VEGF-D-induced aggressive cancer cell phenotype. J Clin Endocrinol Metab 96: E1857-E1861, 2011.

21. Romitti M, Ceolin L, Siqueira DR, Ferreira CV, Wajner SM and Maia AL: Signaling pathways in follicular cell-derived thyroid carcinomas (review). Int J Oncol 42: 19-28, 2013. 
22. Goel HL, Bae D, Pursell B, Gouvin LM, Lu S and Mercurio AM: Neuropilin-2 promotes branching morphogenesis in the mouse mammary gland. Development 138: 2969-2976, 2011.

23. Tai CK, Wang W, Lai YH, Logg CR, Parker WB, Li YF, Hong JS, Sorscher EJ, Chen TC and Kasahara N: Enhanced efficiency of prodrug activation therapy by tumor-selective replicating retrovirus vectors armed with the Escherichia coli purine nucleoside phosphorylase gene. Cancer Gene Ther 17: 614-623, 2010.

24. Liang CC,Park AY and Guan JL: In vitro scratch assay: A convenient and inexpensive method for analysis of cell migration in vitro. Nat Protoc 2: 329-333, 2007.

25. Su JL, Yang PC, Shih JY, Yang CY, Wei LH, Hsieh CY, Chou CH, Jeng YM, Wang MY, Chang KJ, et al: The VEGF-C/Flt-4 axis promotes invasion and metastasis of cancer cells. Cancer Cell 9: 209-223, 2006

26. Issbrücker K, Marti HH, Hippenstiel S, Springmann G, Voswinckel R, Gaumann A, Breier G, Drexler HC, Suttorp N and Clauss M: p38 map kinase - a molecular switch between VEGF-induced angiogenesis and vascular hyperpermeability. FASEB J 17: 262-264, 2003

27. Feng Y, Hu J, Ma J, Feng K, Zhang X, Yang S, Wang W, Zhang J and Zhang Y: RNAi-mediated silencing of VEGF-C inhibits non-small cell lung cancer progression by simultaneously down-regulating the CXCR4, CCR7, VEGFR-2 and VEGFR-3-dependent axes-induced ERK, p38 and AKT signalling pathways. Eur J Cancer 47: 2353-2363, 2011.

28. Affolter A, Fruth K, Brochhausen C, Schmidtmann I, Mann WJ and Brieger J: Activation of mitogen-activated protein kinase extracellular signal-related kinase in head and neck squamous cell carcinomas after irradiation as part of a rescue mechanism. Head Neck 33: 1448-1457, 2011.

29. Hoshida T, Isaka N, Hagendoorn J, di Tomaso E, Chen YL, Pytowski B, Fukumura D, Padera TP and Jain RK: Imaging steps of lymphatic metastasis reveals that vascular endothelial growth factor-C increases metastasis by increasing delivery of cancer cells to lymph nodes: Therapeutic implications. Cancer Res 66 8065-8075, 2006.
30. Goel HL, Pursell B, Chang C, Shaw LM, Mao J, Simin K, Kumar P, Vander Kooi CW, Shultz LD, Greiner DL, et al: GLI1 regulates a novel neuropilin-2/ $\alpha 6 \beta 1$ integrin based autocrine pathway that contributes to breast cancer initiation. EMBO Mol Med 5: 488-508, 2013.

31. Kurio N, Shimo T, Fukazawa T, Takaoka M, Okui T, Hassan NM, Honami T, Hatakeyama S, Ikeda M, Naomoto Y and Sasaki A: Anti-tumor effect in human breast cancer by TAE226, a dual inhibitor for FAK and IGF-IR in vitro and in vivo. Exp Cell Res 317: 1134-1146, 2011.

32. Liu J and Brown RE: Morphoproteomic confirmation of an activated nuclear factor-KBp65 pathway in follicular thyroid carcinoma. Int J Clin Exp Pathol 5: 216-223, 2012.

33. Liu W, Parikh AA, Stoeltzing O, Fan F, McCarty MF, Wey J, Hicklin DJ and Ellis LM: Upregulation of neuropilin-1 by basic fibroblast growth factor enhances vascular smooth muscle cell migration in response to VEGF. Cytokine 32: 206-212, 2005

34. Wang Z, Castresana MR and Newman WH: Reactive oxygen and NF-kappaB in VEGF-induced migration of human vascular smooth muscle cells. Biochem Biophys Res Commun 285: 669-674, 2001.

35. Hall-Jackson CA, Goedert M, Hedge P and Cohen P: Effect of SB 203580 on the activity of c-Raf in vitro and in vivo. Oncogene 18: 2047-2054, 1999.

36. Hu P, Liu W, Wang L, Yang M and Du J: High circulating VEGF level predicts poor overall survival in lung cancer. J Cancer Res Clin Oncol 139: 1157-1167, 2013

37. Zhang Y, Meng X, Zeng H, Guan Y, Zhang Q, Guo S, Liu X and Guo Q: Serum vascular endothelial growth factor-C levels: A possible diagnostic marker for lymph node metastasis in patients with primary non-small cell lung cancer. Oncol Lett 6: 545-549, 2013. 